\title{
Mortalidad de pacientes con infección severa por SARS - CoV2 en ventilación mecánica de una unidad de cuidados intensivos de un hospital general de Lima
}

Mortality of patients with severe COVID-19 during mechanical ventilation in an intensive care unit of a general hospital in Lima

\author{
Diana Fernández Merjildo 1,a; 2 (D), Lady Lévano Díaz 1,a (D), Javier Cieza Zevallos 2,a,b (iD), Jaime Zegarra \\ Piérola ${ }^{1, c ;} 2$ (iD
}

\section{RESUMEN}

Objetivo: Estimar la mortalidad y describir las intervenciones terapéuticas en pacientes con infección severa por SARS CoV-2 en ventilación mecánica de una unidad de cuidados intensivos. Material y métodos: Estudio de cohorte analítica retrospectiva. Se incluyeron 105 pacientes con infección severa por SARS - CoV2 en ventilación mecánica invasiva que ingresaron con el diagnóstico de insuficiencia respiratoria aguda a la unidad de cuidados intensivos del Hospital Cayetano Heredia de marzo a octubre del 2020. Resultados: La mortalidad global de los pacientes en ventilación mecánica invasiva fue 38\%; el 79\% fueron varones, la edad promedio fue 49,8 \pm 13 años, el $65 \%$ no tuvo comorbilidades, el APACHE II fue $12 \pm 6$, el SOFA $5 \pm 3$; cursaron con linfopenia; con niveles séricos de ferritina, deshidrogenasa láctica y proteína $\mathrm{C}$ reactiva altas, el 30\% recibió hidroxicloroquina, el 32\% azitromicina, el $47 \%$ ceftriaxona y el $27 \%$ hidroxicloroquina más azitromicina. La mortalidad fue significativamente mayor en varones $(\mathrm{p}=0,03)$, mayores de 50 años $(\mathrm{p}=0,004)$, con puntaje APACHE II $(\mathrm{p}=0,005)$ y SOFA $(\mathrm{p}=0,0009)$ altos, y con el uso de hidroxicloroquina $(\mathrm{p}=0,001)$, azitromicina $(\mathrm{p}=0,03)$, e hidroxicloroquina más azitromicina $(\mathrm{p}=0,001)$; no hubo diferencia significativa con el uso de corticoides e ivermectina. Conclusiones: La mortalidad en los pacientes con infección severa por SARS - CoV2 en ventilación mecánica invasiva fue significadamente mayor en varones mayores de 50 años, con puntaje APACHE II y SOFA altos, con el uso de hidroxicloroquina, azitromicina y la combinación de los mismos.

PALABRAS CLAVE: Síndrome respiratorio agudo grave, infecciones por coronavirus, mortalidad, respiración artificial. (Fuente: DeCS BIREME).

\section{SUMMARY}

Objective: To report on mortality and therapeutic interventions of patients with severe COVID-19 on mechanical ventilation in an intensive care unit (ICU). Methods: A retrospective cohort study of 105 patients with severe COVID-19 admitted to the ICU with respiratory failure needing mechanical ventilation from march to October

\footnotetext{
Servicio de Cuidados Intensivos Generales. Hospital Cayetano Heredia. Lima, Perú.

Universidad Peruana Cayetano Heredia. Lima, Perú.

Medico Intensivista;

Profesor emérito;

Médico Internista;
} 
2020 was carried-out. Results: overall mortality of patients on mechanical ventilation was $38 \%$; $79 \%$ were males: mean age was $49.8 \pm 13$ years; $65 \%$ did not have comorbidities; APACHE II score was $12 \pm 6$; and the SOFA score was $5 \pm 3$. Patients presented with lymphopenia, high serum levels of ferritin, lactic dehydrogenase and C-reactive protein. Thirty percent received hydroxychloroquine, $32 \%$ received azithromycin, $47 \%$ ceftriaxone and $27 \%$ received hydroxychloroquine plus azithromycin. Mortality was significantly higher in males $(\mathrm{p}=0,004)$, with high APACHE II $(\mathrm{p}=0.005)$ and high SOFA $(\mathrm{p}=0.0009)$ scores and with use of hydroxychloroquine $(\mathrm{p}=0,001)$, azithromycin ( $\mathrm{p}=0.03)$ and both hydroxychloroquine plus azithromycin $(\mathrm{p}=0.001)$, no difference was observed with the use of steroids and ivermectin. Conclusions: The mortality of patients with severe COVID-19 admitted to the ICU on mechanical ventilation was higher in males and in those who received hydroxychloroquine, azithromycin or hydroxychloroquine plus azithromycin.

KEYWORDS: Severe acute respiratory syndrome, coronavirus infections, mortality, respiration, artificial. (Source: MeSH NLM).

\section{INTRODUCCIÓN}

La actual pandemia de la COVID-19 se originó en Wuhan, provincia de Hubei, China, en diciembre de 2019. El agente etiológico es un nuevo coronavirus denominado SARS-CoV-2 con similitud estructural con los virus responsables del síndrome respiratorio agudo severo (SARS-CoV) y del Medio Oriente $(\mathrm{MERS}-\mathrm{CoV})^{(1)}$.

Los casos severos de infección por SARS-CoV-2 pueden presentar neumonía, síndrome respiratorio agudo severo (SDRA), coagulopatía, insuficiencia renal, falla orgánica múltiple e incluso fallecer. Los pacientes que ingresan a las unidades de cuidados intensivos (UCI) suelen requerir ventilación mecánica invasiva. La edad avanzada y puntuaciones altas en la escala de evaluación secuencial de falla orgánica (SOFA) se asociaron con mayor probabilidad de muerte ${ }^{(2)}$.

En Perú, el diagnóstico de COVID-19 se realiza mediante pruebas moleculares (RT- PCR) en muestras oro faríngeas y nasofaríngeas ${ }^{(3)}, \mathrm{y}$ con pruebas rápidas para la detección de anticuerpos IgM e IgG contra SARS-CoV-2 en muestras de sangre, suero o plasma (4). El hallazgo de laboratorio más común descrito en pacientes con la COVID-19 fue linfopenia con valores absolutos $<1,000$ células $/ \mathrm{mm}^{3}$; niveles séricos incrementados de lactato deshidrogenasa (LDH) ${ }^{(5)}$, así mismo, el incremento de niveles séricos de LDH, dimero-D y fibrinógeno fueron indicadores útiles de pronóstico y severidad de enfermedad por COVID-19 (6). La proteína C reactiva (PCR) se incrementó en el 75 93\% de pacientes con infección severa, la disminución de niveles séricos de albúmina se relacionó con una mayor frecuencia de malos resultados ${ }^{(7)}$.
La infección por SARS-CoV-2 se caracteriza por un alto contagio, alta morbilidad y mortalidad, pero hasta el momento no se han desarrollado medicamentos específicos para su tratamiento. Los investigadores tratan de encontrar objetivos terapéuticos del virus para desarrollar medicamentos dirigidos de alta eficacia y baja toxicidad ${ }^{(8)}$. En vista de que la información publicada a la fecha sobre las estrategias de manejo en pacientes críticamente enfermos con infección por SARS-CoV-2 no han mostrado evidencia sólida, es necesario observar y describir el comportamiento de los pacientes en el ámbito local, así como las estrategias de terapia utilizadas y su impacto clínico.

El estudio tuvo como objetivo estimar la mortalidad y describir las intervenciones terapéuticas en pacientes con infección severa por SARS CoV-2 en ventilación mecánica de una unidad de cuidados intensivos.

\section{MATERIAL Y MÉTODOS}

\section{Diseño del estudio y participantes}

Estudio descriptivo de cohorte retrospectiva realizado en la unidad de cuidados intensivos (UCI) de Hospital Cayetano Heredia, en el periodo comprendido entre el 15 de marzo y el 7 de octubre del 2020. Se incluyeron a pacientes mayores de 18 años de edad con diagnóstico de infección por SARS - CoV2 definido por reacción de cadena de la polimerasa en tiempo real (PCR - RT) o prueba antigénica, que ingresaron a ventilación mecánica invasiva en la UCI. Se excluyeron a gestantes.

Los pacientes con infección severa por SARS CoV2 cursaron con síndrome de dificultad respiratoria aguda, caracterizado por edema pulmonar de origen no cardiogénico de presentación aguda menor a 7 días, 
con evidencia hipoxemia refractaria $\mathrm{Pa} 02 / \mathrm{Fi} 02<300$, con necesidad de intubación y ventilación mecánica invasiva; con insuficiencia cardiaca aguda secundaria a carditis; injuria renal aguda con evidencia de oliguria y elevación de la creatinina $>1,5 \mathrm{mg} / \mathrm{dl}$ y elevación $>0,3 \mathrm{mg} / \mathrm{dl} / \mathrm{día}$; disfunción neurológica caracterizado por score de coma de Glasgow menor de 12, y con diagnóstico definitivo por PCR - RT o prueba antigénica y sospecha de infección por SARS - CoV2.

\section{Colección de datos}

Los datos epidemiológicos, clínicos, tratamiento, monitoreo en UCI se obtuvieron de los registros electrónicos de la UCI. Se usó la historia clínica electrónica consignada como Shegueo2, donde se encuentran los datos de filiación, anamnesis, examen físico, diagnóstico, evolución y epicrisis; las indicaciones médicas día por día estuvieron consignadas en un archivo Drive consignada como UCICORONITA, las mismas fueron revisadas y sirvieron para la obtención de las indicaciones médicas.
La data de laboratorio estuvo consignada como Labcore para la revisión y obtención de datos de laboratorio; asimismo se usaron los registros electrónicos de enfermería. Los datos fueron obtenidos por tres investigadores asignados como (DFM, JZP y LLD).

\section{Procedimientos}

Los pacientes ingresaron a la UCI procedentes de otras áreas del hospital habilitadas para atención de pacientes con sospecha y diagnóstico confirmado de SARS - CoV2; dichas áreas fueron identificadas como triaje de pacientes con COVID 19, CENEX, Medicina Tropical, Medicina pabellón B, Unidad de Shock Trauma emergencia, área de observación de emergencia, los pacientes ingresaron a UCI previa coordinación.

\section{Análisis estadístico}

Se utilizó estadística descriptiva en la población de estudio según sus características clínicas. Para las variables cuantitativas se usó medias y desviaciones

Tabla 1. Características basales de los pacientes incluidos en el estudio

\begin{tabular}{lccc}
\hline \multicolumn{1}{c}{ CARACTERISTICA } & Mujer & Varón & Total \\
\hline Edad (años) media \pm DE & $43,36 \pm 13,5$ & $51,48 \pm 12,58$ & $49,78 \pm 13.14$ \\
APACHE II (puntaje) media \pm DE & $12,18 \pm 4.45$ & $12,19 \pm 6,27$ & $12,19 \pm 5,93$ \\
SOFA (Puntaje) media \pm DE & $4,32 \pm 1,86$ & $5,52 \pm 2,82$ & $5,26 \pm 2,68$ \\
INDICE DE MASA CORPORAL N (\%) & & & \\
Normal (IMC $\left.<25 \mathrm{~kg} / \mathrm{m}^{2}\right)$ & $1(4,55 \%)$ & $6(7,23 \%)$ & $7(6,67 \%)$ \\
Sobrepeso (IMC $\left.25-30 \mathrm{~kg} / \mathrm{m}^{2}\right)$ & $4(18,18 \%)$ & $38(45,78 \%)$ & $42(40,0 \%)$ \\
Obesidad (IMC $\left.>30 \mathrm{~kg} / \mathrm{m}^{2}\right)$ & $17(77,27 \%)$ & $39(46,99 \%)$ & $56(53,33 \%)$ \\
ANTECEDENTES N (\%) & & & \\
No antecedentes & $18(81,82 \%)$ & $50(60,24 \%)$ & $68(64,76 \%)$ \\
Hipertensión arterial & $2(9,09 \%)$ & $15(18,07 \%)$ & $17(16,19 \%)$ \\
Diabetes mellitus tipo 2 & $2(9,09 \%)$ & $10(12,05 \%)$ & $12(11,43 \%)$ \\
Tuberculosis pulmonar & $0(0,0 \%)$ & $2(2,41 \%)$ & $2(1,90 \%)$ \\
Arritmia cardiaca & $0(0,00 \%)$ & $4(4,82 \%)$ & $4(3,81 \%)$ \\
Asma bronquial & $0(0,00 \%)$ & $2(2,41 \%)$ & $2(1,90 \%)$ \\
LABORATORIO $(\mathbf{m e d i a} \pm$ DE) & & & $863,33 \pm 679,38$ \\
Linfocitos (células/mm $)$ & $820,74 \pm 441,62$ & $874,91 \pm 732,57$ & $1298,28 \pm 1041,04$ \\
Ferritina (ug/l) & $419,92 \pm 453,83$ & $1489,93 \pm 1036,24$ & $569,49 \pm 338,26$ \\
DHL (UI/l) & $603,36 \pm 491,12$ & $559,94 \pm 284,45$ & $168,65 \pm 197,38$ \\
Proteína C reactiva (mg/dl) & $129,14 \pm 98,90$ & $179,66 \pm 221,22$ & $2,67 \pm 0,55$ \\
Albumina (gr/dl) & $2,69 \pm 0,53$ & $2,67 \pm 0,55$ & \\
\hline
\end{tabular}

SOFA: Evaluación secuencial de falla de órganos, APACHE: Evaluación de salud crónica y fisiología aguda 
estándar, medianas y rangos intercuartílico dependiendo de si las variables tenían o no distribución normal. Para las variables categóricas se utilizó frecuencias y porcentajes.

Para determinar la asociación de variables cuantitativas a mortalidad en el análisis bivariado se empleó la prueba t de student o la suma de rangos de Wilcoxon dependiendo de la distribución de la variable. En el caso de comparación de variables cualitativas se utilizó la prueba exacta de Fisher o la prueba de chi cuadrado para tendencia lineal según correspondió. Finalmente se realizó el análisis multivariado utilizando ecuaciones estimadoras generalizadas (acrónimo GLM en inglés) para las variables con $\mathrm{p}<0,05$ y regresión de Cox para comparar tiempo hasta muerte. Se consideró significancia estadística con $\mathrm{p}<$ 0,05 . El análisis se realizó con el programa estadístico Stata versión 17 (número de serie 401709310706; Licencia Universidad Peruana Cayetano Heredia).

El estudio fue evaluado y aprobado por el comité de ética del Hospital Cayetano Heredia.

\section{RESULTADOS}

El estudio incluyó 105 pacientes con infección severa por SARS-CoV2, con insuficiencia respiratoria aguda hipoxémica en ventilación mecánica. La edad promedio de los pacientes fue $49.8 \pm 13$ años, el $79 \%$ fueron varones, el $53 \%$ tuvo obesidad, el $65 \%$ no tuvo antecedentes patológicos, el 16\%. Al ingreso, las puntuaciones promedio de las escalas de evaluación de salud crónica y fisiología aguda (APACHE II) y de evaluación secuencial de falla de órganos (SOFA) fueron $12 \pm 6$ y $5 \pm 3$ puntos, respectivamente. Los exámenes de laboratorio mostraron linfopenia severa, ferritina elevada, deshidrogenasa láctica elevada, proteína $\mathrm{C}$ reactiva alta e hipoalbuminemia, tal como se muestra en la tabla 1.

El $30 \%$ recibió hidroxicloroquina, el $32 \%$ azitromicina, el $47 \%$ ceftriaxona, el $27 \%$ hidroxicloroquina más azitromicina, el $28 \%$ ivermectina, el $30 \%$ corticoides, como metilprednisolona, hidrocortisona o dexametasona (tabla 2). Para las infecciones bacterianas nosocomiales recibieron meropenem, colistina, piperacilina/ tazobactam, vancomicina.

La mortalidad fue significativamente mayor en pacientes de sexo masculino $(\mathrm{p}=0,03)$, mayores de 50 años $(p=0,004)$, con score APACHE II $(p=0,005)$ y SOFA ( $p=0,0009)$ con puntaje alto; con ferritina, deshidrogenasa láctica altas ehipoalbuminemia (gráfico 1). Los pacientes que recibieron hidroxicloroquina $(\mathrm{p}=0,001)$, azitromicina $(\mathrm{p}=0,03)$, hidroxicloroquina más azitromicina $(\mathrm{p}=0,001)$, hidroxicloroquina más ceftriaxona $(\mathrm{p}=0,005)$, hidroxicloroquina más azitromicina más ceftriaxona $(\mathrm{p}=0,005)$, tuvieron una mortalidad significativamente mayor que los que no recibieron (tabla 3 , gráficos 2,3 y 4 ).

La mortalidad global fue $38 \%$, el tiempo de permanencia en la unidad de cuidados intensivos (UCI) fue $17 \pm 12$ días; 62 (59\%) pacientes desarrollaron una infección intrahospitalaria, 41 (39\%) tuvieron neumonía asociada al ventilador mecánico teniendo como gérmenes más frecuentes a Acinetobacter baumani y Pseudomona aeruginosa, 5 (4,76\%)

Tabla 2. Características de las intervenciones iniciales en los pacientes.

\begin{tabular}{lccc}
\hline \multirow{2}{*}{ INTERVENCIONES } & Mujer & Varón & Total \\
\cline { 2 - 4 } & $\mathbf{n ~ ( \% )}$ & $\mathbf{n ~ ( \% )}$ & $\mathbf{n}(\mathbf{\%})$ \\
\hline Hidroxicloroquina & $7(31,82 \%)$ & $24(28,92 \%)$ & $31(29.52 \%)$ \\
Azitromicina & $10(45,45 \%)$ & $24(28,92 \%)$ & $34(32,38 \%)$ \\
Ceftriaxona & $14(63,64 \%)$ & $35(42,17 \%)$ & $49(46,67 \%)$ \\
Hidroxicloroquina + Azitromicina & $7(311,82 \%)$ & $21(25,30 \%)$ & $28(26,67 \%)$ \\
Hidroxicloroquina + ceftriaxona & $7(31,82 \%)$ & $19(22,89 \%)$ & $26(24,76 \%)$ \\
Hidroxicloroquina + azitromicina + ceftriaxona & $7(31,82 \%)$ & $19(22,89 \%)$ & $16(24,76 \%)$ \\
Corticoides & $6(27,27 \%)$ & $25(30,12 \%)$ & $31(29,52 \%)$ \\
Ivermectina & $8(36,36 \%)$ & $21(25,30 \%)$ & $29(27,62 \%)$ \\
\hline
\end{tabular}



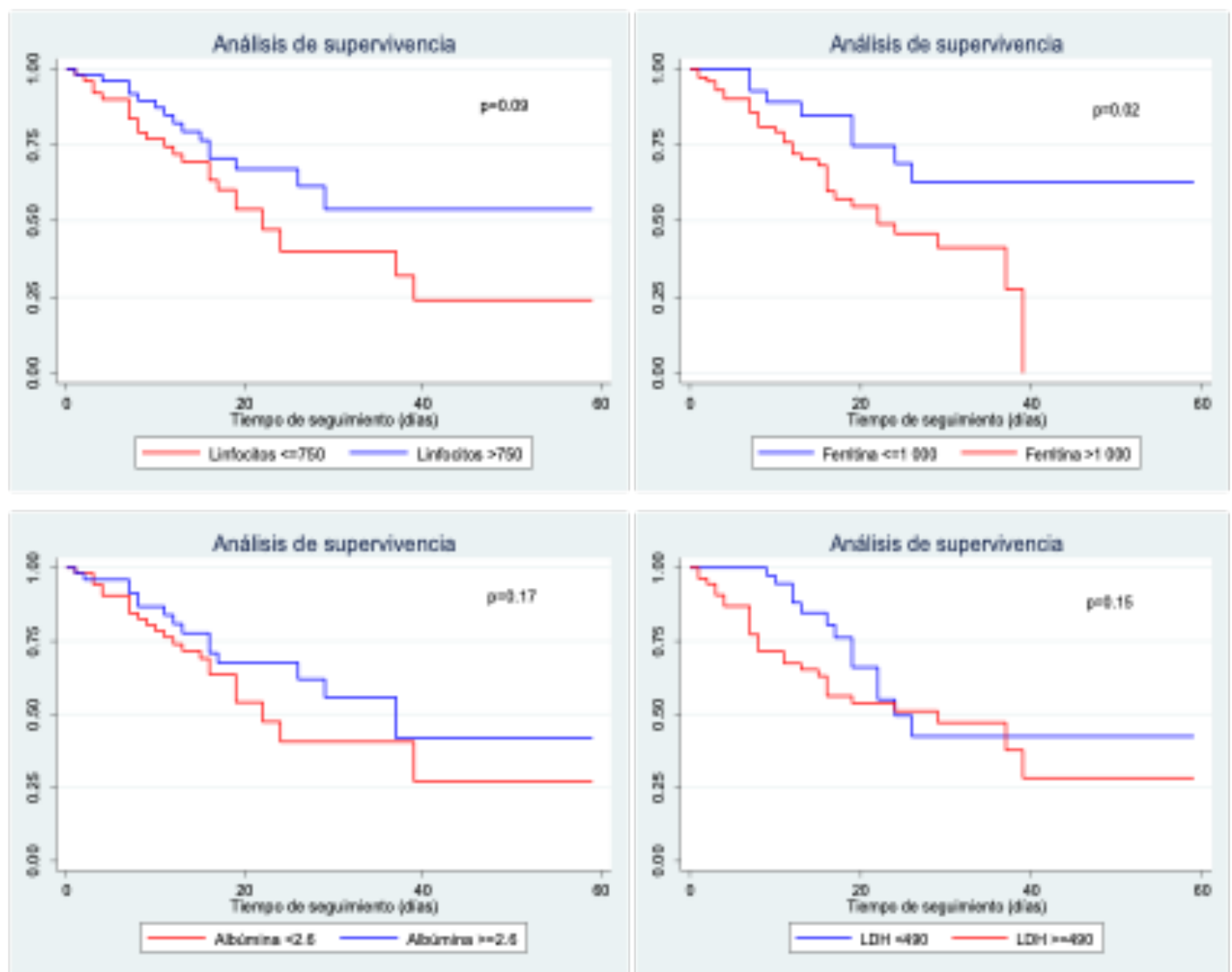

Gráfico 1. Análisis de supervivencia al día 60 en relación a los hallazgos de laboratorio al ingreso a la

UCI.

desarrollaron bacteriemia por Klebsiella pneumoniae y $16(15,23 \%)$ neumonía asociada al ventilador mecánico más bacteriemia.

\section{DISCUSIÓN}

En el estudio se incluyeron 105 pacientes con infección confirmada por SARS - CoV2 en ventilación mecánica invasiva en la unidad de cuidados intensivos; la mortalidad global fue $38 \%$, comparable a la mortalidad encontrada por Gupta et al. ${ }^{(9)}$, quienes encontraron $35,4 \%$ y Auld et al. ${ }^{(10)}$, en Atlanta $35,7 \%$, e inferior a la encontrada por Rahim et al. ${ }^{(11)}$, en Pakistan (77\%), Richardson et al. ${ }^{(12)}$, en New York $(75,6 \%)$, Arentz et al. ${ }^{(13)}$, en Washington (67\%), y Yang et al. ${ }^{(14)}, 61,5 \%$ en China; sin embargo, la mortalidad fue menor en la reportada por Mitra et al. ${ }^{(15)}$, en Canadá $15,4 \%$, Lee et al. ${ }^{(16)}$, en Corea $20,4 \%$ y Grasselli et al. ${ }^{(17)}$, en Italia $26 \%$. Las razones para la diferencia en la mortalidad en los diferentes países, podría estar relacionada con la preparación de los sistemas de salud, la presencia de un staff médico especializado en las unidades de cuidados intensivos, equipamiento y recursos en las mismas.
La edad promedio de los pacientes fue 49,8 \pm 13 , menor que la encontrada en los estudios publicados en Italia, EE UU, Canadá, China donde la edad promedio estuvo en el rango entre 57 y 73 años. La menor edad de nuestra población estaría relacionada a la mayor exposición epidemiológica por ser la económicamente activa. El $79 \%$ fueron varones, similar a las series publicadas en otros países, con excepción del trabajo publicado por Lee ${ }^{(16)}$, donde el $55,1 \%$ fueron mujeres.

Se ha reportado que las comorbilidades son factores de riesgo para enfermedad severa por SARS - CoV2; sin embargo, varía en los diferentes países en el mundo. El 65\% de nuestros pacientes críticos con infección por SARS - CoV2 no tenía antecedentes de enfermedades previas, el 16\% hipertensión arterial, $11 \%$ diabetes mellitus, hallazgos similares a los publicados por Rahim ${ }^{(11)}$ en Pakistan, pero diferente a los estudios publicados en EEUU, China, Italia, donde la hipertensión arterial, enfermedad cardiovascular y diabetes fueron las comorbilidades más frecuentes variando entre 30 y $40 \%$ de los pacientes. 
Tabla 3. Mortalidad relacionada a características clínicas, de laboratorio y terapéuticas.

\begin{tabular}{|c|c|c|c|}
\hline Características clínicas & Vivo & Falleció & $\mathbf{p}$ \\
\hline Edad (años) & $47 \pm 2$ & $54 \pm 2$ & 0,00 \\
\hline Sexo masculino & & & 0,03 \\
\hline Escala SOFA (puntaje) & $4,5 \pm 2$ & $6,5 \pm 3$ & 0,01 \\
\hline Escala APACHE II (puntaje) & $10,9 \pm 0,7$ & $14,4 \pm 1$ & 0,01 \\
\hline Tiempo en UCI (días) & $18,4 \pm 13$ & $13,6 \pm 9$ & 0,06 \\
\hline \multicolumn{4}{|l|}{ Características de Laboratorio } \\
\hline Linfocitos (células $/ \mathrm{mm}^{3}$ ) & $908 \pm 752$ & $788 \pm 538$ & 0,21 \\
\hline Ferritina (ug/l) & $1159 \pm 1111$ & $1516 \pm 896$ & 0,04 \\
\hline DHL UI/1 & $513 \pm 314$ & $668 \pm 360$ & 0,01 \\
\hline PCR mg/l & $154 \pm 213$ & $190 \pm 169$ & 0,17 \\
\hline Albumina gr/dl & $2,7 \pm 0,5$ & $2,5 \pm 0,4$ & 0,01 \\
\hline \multicolumn{4}{|l|}{ Características Terapéuticas } \\
\hline Hidroxicloroquina & $11(17 \%)$ & $20(50 \%)$ & 0,001 \\
\hline Azitromicina & $16(25 \%)$ & $18(45 \%)$ & 0,03 \\
\hline Ceftriaxona & $28(43 \%)$ & $21(53 \%)$ & 0,34 \\
\hline Hidroxicloroquina + Azitromicina & $10(15 \%)$ & $18(45 \%)$ & 0,01 \\
\hline Hidroxicloroquina + Ceftriaxona & $10(45 \%)$ & $16(40 \%)$ & 0,01 \\
\hline Corticoides & $15(23 \%)$ & $16(40 \%)$ & 0,06 \\
\hline Ivermectina & $17(26 \%)$ & $12(30 \%)$ & 0,66 \\
\hline \multicolumn{4}{|l|}{ Infecciones nosocomiales/ terapéutica } \\
\hline Infecciones nosocomiales & $42(62 \%)$ & $20(50 \%)$ & 0,24 \\
\hline \multicolumn{4}{|l|}{ Terapéutica } \\
\hline Piperacilina- Tazobactam & $22(34 \%)$ & $12(30 \%)$ & 0,68 \\
\hline Meropenem & $41(63 \%)$ & $26(65 \%)$ & 0,84 \\
\hline Vancomicina & $16(25 \%)$ & $15(38 \%)$ & 0,16 \\
\hline Colistina & $30(46 \%)$ & $16(40 \%)$ & 0,53 \\
\hline
\end{tabular}

El 53\% de nuestros pacientes tuvo el diagnóstico de obesidad; considerado como un factor de riesgo para enfermedad severa, sin embargo, no es reportado en muchos de los estudios publicados de pacientes críticos con SARS - CoV2 en cuidados intensivos.

La evaluación de enfermedad critica por medio de las escalas de salud crónica y fisiológica aguda (APACHE II) y de evaluación secuencial de falla de órganos (SOFA) fue similar al de los estudios publicados por Yang ${ }^{(14)}$ en China y Mitra ${ }^{(15)}$ en
Canadá; los pacientes con mayor puntaje en dichas escalas tuvieron mortalidad significativamente mayor.

Los marcadores inflamatorios como ferritina sérica, proteína $C$ reactivay de daño celulartipo deshidrogenasa láctica (DHL), fueron significativamente mayores en los pacientes que fallecieron, similar a lo publicado por Mitra en Canadá ${ }^{(15)}$.

La mortalidad fue significativamente mayor en los pacientes que usaron hidroxicloroquina, azitromicina; 


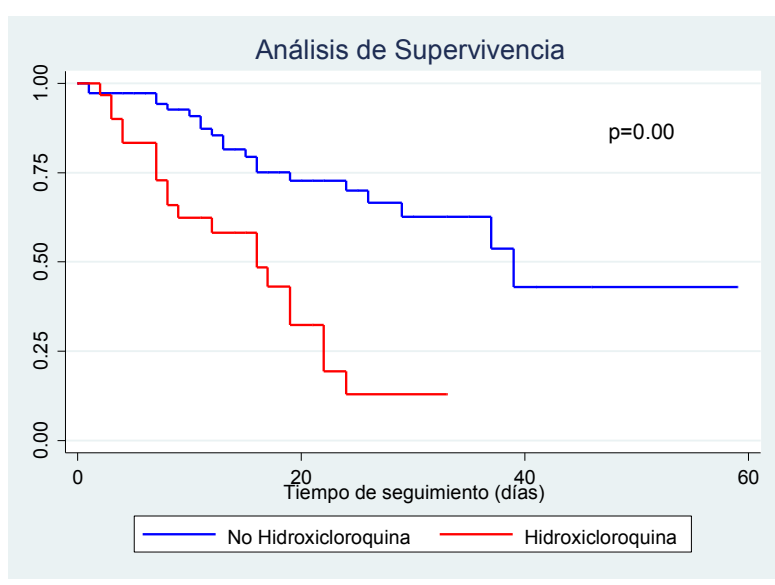

Gráfico 2. Análisis de supervivencia al día 60 en pacientes que recibieron hidroxicloroquina.

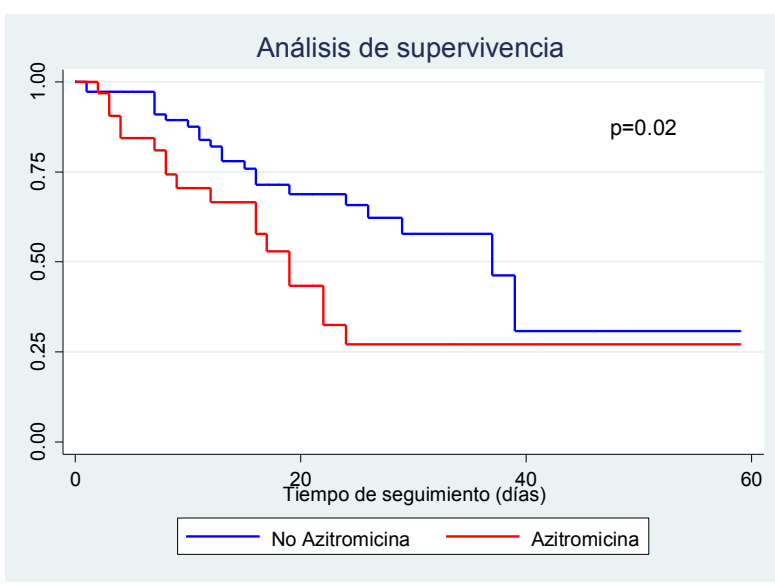

Gráfico 3. Análisis de supervivencia al día 60 en pacientes que recibieron azitromicina.

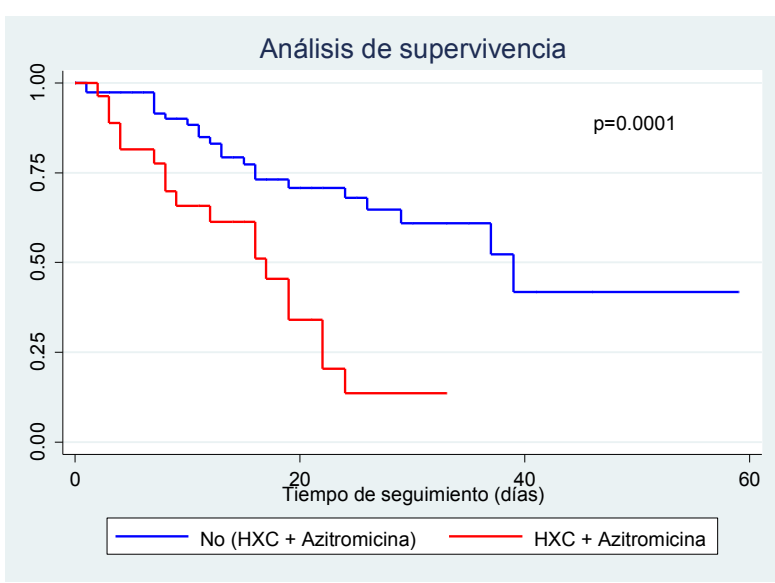

Gráfico 4. Análisis de supervivencia al día 60 de los pacientes que recibieron la combina hidroxicloroquina + azitromicina. hidroxicloroquina más azitromicina, hidroxicloroquina más azitromicina más ceftriaxona, comparado con quienes no usaron. Los estudios publicados por Horby en el Reino Unido, Geleris en New York y Self de Oxford, mostraron que la administración de hidroxicloroquina no mejoró el estatus clínico ni disminuyó la mortalidad a los 28 días, comparado con el tratamiento usual ${ }^{(18,19,20,21)}$ por lo cual se desestima su uso; Furtado ${ }^{(22)}$ en Brasil, no encontró diferencia en la mortalidad entre azitromicina asociada al tratamiento usual versus el control.

En el tratamiento de infecciones bacterianas secundarias nosocomiales se usó meropenem, piperacilina/tazobactam, colistina, vancomicina, sin evidencia de diferencia significativa en mortalidad entre sobrevivientes y no sobrevivientes; similar a las publicaciones de Canadá, EE UU, China.

No se encontró diferencia significativa en la mortalidad con el uso de corticoides entre sobrevivientes y no sobrevivientes $(\mathrm{p}=0,06)$, a diferencia de los hallazgos de Horby y su grupo de trabajo en el Reino Unido, quienes encontraron que la dexametasona disminuía la mortalidad en pacientes hospitalizados con requerimiento de oxígeno y en pacientes críticos con SARS - CoV2 ${ }^{(23)}$; esto podría explicarse por qué el presente estudio fue observacional, en el cual solo el 30\% uso corticoide. De igual manera no se encontró diferencia en la mortalidad con el uso de ivermectina.

Las limitaciones de nuestro estudio están relacionadas al diseño del mismo, la cual estaría propensa a sesgos y a la falta de información completa.

Concluimos que la mortalidad en los pacientes con infección severa por SARS - COV2 en ventilación mecánica invasiva fue significadamente mayor en varones mayores de 50 años, con puntaje APACHE II y SOFA altos; uso de hidroxicloroquina, azitromicina y la combinación del mismos con ceftriaxona.

\section{Agradecimientos:}

Al Dr. José Ticona, Dr. Miguel Ramírez y Dr. Jorge Córdova Segil.

\section{Declaración de financiamiento y de conflictos de interés:}

El estudio fue financiado por los autores. No existen conflictos de intereses de los autores de orden económico, institucional, laboral o personal. 


\section{Contribución de autoría:}

DFM: Participó en la concepción y diseño del estudio, recolección e interpretación de datos, redacción del artículo, así como en la aprobación de la versión final. JZP: Participó en la concepción del estudio, recolección e interpretación de datos, así como en la revisión crítica del articulo y aprobación de la versión final. LLD: Participó en la recolección e interpretación de datos, así como en la revisión crítica del artículo y aprobación de la versión final. JCZ: Participó en el análisis e interpretación de datos, así como en la aprobación de la versión final.

\section{Correspondencia:}

Diana Fernández Merjildo

Correo electrónico: diana.fernandez.m@upch.pe

\section{REFERENCIAS BIBLIOGRÁFICAS}

1. Phelan AL, Katz R, Gostin LO. The Novel Coronavirus Originating in Wuhan, China: Challenges for Global Health Governance. JAMA. 2020; 323(8):709-710. doi: 10.1001/jama.2020.1097

2. Zhou F, Yu T, Du R, et al. Clinical course and risk factors for mortality of adult inpatients with COVID-19 in Wuhan, China: a retrospective cohort study. Lancet. 2020; 395(10229):1054-1062. doi: 10.1016/S0140-6736(20)30566-3

3. Corman VM, Landt O, Kaiser M, et al. Detection of 2019 novel coronavirus $(2019-\mathrm{nCoV})$ by realtime RT-PCR. Euro Surveill. 2020; 25(3):2000045. doi: 10.2807/1560-7917.ES.2020.25.3.2000045

4. Instituto Nacional de Salud. Uso de pruebas rápidas para COVID-19. Lima: Instituto Nacional de Salud; 2021. https://web.ins.gob.pe/

5. Fan BE, Chong VCL, Chan SSW, et al. Hematologic parameters in patients with COVID-19 infection. Am J Hematol. 2020; 95(6): E131-E134. doi: 10.1002/ajh. 25774

6. Tang N, Li D, Wang X, Sun Z. Abnormal coagulation parameters are associated with poor prognosis in patients with novel coronavirus pneumonia. J Thromb Haemost. 2020; 18(4):844-7.

7. Frater JL, Zini G, d'Onofrio G, Rogers HJ. COVID-19 and the clinical hematology laboratory. Int J Lab Hematol. 2020; 42 Suppl 1:11-18. doi: 10.1111/ijlh.13229

8. Kang S, Peng W, Zhu Y, et al. Recent progress in understanding 2019 novel coronavirus (SARSCoV-2) associated with human respiratory disease: detection, mechanisms and treatment. Int $\mathbf{J}$ Antimicrob Agents. 2020; 55(5):105950. doi: 10.1016/j.ijantimicag.2020.105950
9. Gupta S, Hayek S, Wang W, et al. Factors Associated with Death in Critically Ill Patients with Coronavirus Disease 2019 in the US. JAMA Intern Med. 2020; 180(11):1436-1447. doi: 10.1001/ jamainternmed.2020.3596

10. Auld S, Caridi-Scheible M, Blum J, et al. ICU and Ventilator Mortality Among Critically Ill Adults with Coronavirus Disease 2019. Crit Care Med. 2020; 48(9):e799-e804. doi: 10.1097/CCM.00000 00000004457

11. Rahim F, Amin S, Noor M, et al. Mortality of patients with severe COVID-19 in the Intensive Care Unit: An observational study from a major COVID-19 Receiving Hospital. Cureus. 2020; 12(10): e10906. DOI 10.7759/cureus.10906

12. Richardson S, Hirsch J, Narasimhan $M$, et al. Presenting Characteristics, Comorbidities, and Outcomes Among 5700 Patients Hospitalized With COVID-19 in the New York City Area. JAMA. 2020; 323(20):2052-2059. doi:10.1001/jama.2020.6775

13. Arentz M, Yim E, Klaff L, et al. Characteristics and Outcomes of 21 Critically Ill Patients With COVID-19 in Washington State. JAMA. 2020; 323(16):16121614. doi: 10.1001/jama.2020.4326

14. Yang X, Yu Y, Xu J, et al. Clinical course and outcomes of critically ill patients with SARS-CoV-2 pneumonia in Wuhan, China: a single-centered, retrospective, observational study. Lancet Respir Med. 2020; 8(5):475-481. doi: 10.1016/S2213-2600(20)30079

15. Mitra A, Fergusson N, Lloyd-Smith E, et al. Baseline characteristics and outcomes of patients with COVID-19 admitted to intensive care units in Vancouver, Canada: a case series. CMAJ. 2020; 192: E694-701. doi: 10.1503/cmaj.200794.

16. Lee JY, Kim HA, Huh K, et al. Risk Factors for Mortality and Respiratory Support in Elderly Patients Hospitalized with COVID-19 in Korea. J Korean Med Sci. 2020; 35(23):e223. doi: 10.3346/ jkms.2020.35.e223

17. Grasselli G, Zangrillo A, Zanella A, et al. Baseline Characteristics and Outcomes of 1591 Patients Infected With SARS-CoV-2 Admitted to ICUs of the Lombardy Region, Italy. JAMA. 2020; 323(16):15741581. doi:10.1001/jama.2020.5394.

18. RECOVERY Collaborative Group; Horby P, Mafham $M$, et al. Effect of Hydroxychloroquine in Hospitalized Patients with Covid-19. N Engl J Med. 2020; 383(21):2030-2040. doi: 10.1056/ NEJMoa2022926.

19. Cavalcanti A, Zampieri F, Rosa R, et al. Hydroxychloroquine with or without Azithromycin in Mild-to-Moderate Covid-19. N Engl J Med. 2020; 383(21):2041-2052. doi: 10.1056/NEJMoa2019014

20. Geleris J, Sun Y, Platt J, et al. Observational Study of Hydroxychloroquine in Hospitalized Patients with Covid-19. N Engl J Med 2020; 382:2411-8. 
DOI: 10.1056/NEJMoa2012410

21. Self W, Semler M, Leither L, et al. Effect of Hydroxychloroquine on Clinical Status at 14 Days in Hospitalized Patients With COVID-19. A Randomized Clinical Trial. JAMA. 2020; 324(21):2165-2176. doi: 10.1001/jama.2020.22240

22. Furtado R, Berwanger O, Fonseca $H$, et al. Azithromycin in addition to standard of care versus standard of care alone in the treatment of patients admitted to the hospital with severe COVID-19 in
Brazil (COALITION II): a randomised clinical trial. Lancet. 2020; 396(10256):959-967. doi: 10.1016/ S0140-6736(20)31862-6

23. RECOVERY Collaborative Group; Horby P, Lim WS, et al. Dexamethasone in Hospitalized Patients with Covid-19 - Preliminary Report. N Engl J Med. 2021;384(8):693-704. doi: 10.1056/NEJMoa2021436

Recibido: $17 / 05 / 2021$

Aceptado: 23/09/2021 